\title{
Chapter 8 \\ Teaching Tolerance in a Globalized World: Final Remarks
}

\author{
Maria Magdalena Isac, Andrés Sandoval-Hernández \\ and Daniel Miranda
}

\begin{abstract}
Each of the five empirical studies presented in this report aimed to identify factors and conditions that help schools and teachers to promote tolerance in a globalized world. Each study acknowledged the complex, hierarchical layers of explanatory mechanisms, while focusing on what could be learned from in-depth analysis of data collected by the International Association for the Evaluation of Educational Achievement's International Civic and Citizenship Education Study 2009. In this chapter, key findings are summarized, while acknowledging limitations and caveats, and avenues for further research are identified. The report findings also flag some potential implications for policymakers.
\end{abstract}

Keywords Egalitarian attitudes - International Civic and Citizenship Education Study (ICCS) • International large-scale assessments • Tolerance

\subsection{Key Findings}

Each of the five empirical studies presented in this volume aimed to identify factors and conditions that help schools and teachers to promote tolerance in a globalized world. Each of them acknowledged the complex, hierarchical layers of explanatory mechanisms (see Chap. 1, Fig. 1.1), but reported in-depth on a particular topic and level of analysis. Chapter 2 provides a summary of the analytical strategy applied in each of the chapters.

\footnotetext{
M. M. Isac ( $\bowtie)$

University of Groningen, Groningen, The Netherlands

e-mail: mariamagdalena.isac@gmail.com
}

\section{A. Sandoval-Hernández \\ University of Bath, Bath, UK \\ D. Miranda}

Centro de Medición MIDE UC, Pontificia Universidad Católica de Chile, Santiago, Chile 
In Chap. 3, Miranda and Castillo aimed to examine, from a comparative perspective, the reliability and validity of the main constructs used to measure tolerance (attitudes toward equal rights). They applied a broader conceptualization and operationalized definition of tolerance as a set of three, interrelated attitudes toward the rights of women, immigrants and ethnic minorities. They investigated the cross-cultural comparability of these latent variables through empirical analyses of measurement invariance conducted in a factor-analytical framework (confirmatory factor analysis and multigroup confirmatory factor analysis models) applied to the International Civic and Citizenship Education Study (ICCS) 2009 data. By applying multigroup confirmatory factor analyses techniques in order to test the validity of the conceptual framework elaborated upon across the chapters of this book. They found that the three scales used to measure tolerance had the same structure and were equivalent across all the countries analyzed at the scalar level of invariance. By implication, direct comparisons of the mean scores and correlates of the three egalitarian attitudes across countries are empirically justified and can be interpreted in a meaningful way (Desa 2014, Rutkowski and Svetina 2014). Moreover, the analysis also confirmed that multilevel modeling was an adequate strategy for the estimation of the explanatory models. Chapter 3 provides a solid methodological and theoretical basis for all the comparative and multilevel analyses reported in the subsequent chapters, and contributes to the current research by providing empirical support to a broader conceptualization of tolerance based on the ICCS 2009 data.

In what can be considered a general study of factors associated with attitudes toward egalitarian values, Chap. 4 focused on the capacity of schools and other agents to promote positive attitudes toward diversity. Treviño, Béjares, Wyman and Villalobos aimed to analyze how several characteristics of schools and individuals shape student attitudes toward equal rights. Multilevel regression analyses conducted within countries showed both commonalities and differences in the explanatory mechanisms present across countries. The results provided important information that helped to determine the focus of the subsequent chapters. They first identified that individual and family characteristics had a stronger positive influence than schools across all the countries analyzed. Individual student characteristics, such as their interest in social and political issues and their support for democratic values, were related both positively and generally with young peoples' attitudes toward equal rights. In most countries, female students tended to exhibit more positive egalitarian attitudes. Further, and in agreement with previous studies (see, for example, Caro and Schulz 2012), an open classroom climate for discussion and participation in civic-related activities at school were the two school variables that exhibited the most consistent positive relationship with attitudes toward equal rights. This study also revealed the importance of country-specific contexts when examining the relationships between different school variables and the three indicators of tolerance. Different school composition or segregation variables (percentage of girls, immigrants and students belonging to an ethnic minority), and the socioeconomic composition of the school 
population, show strong differential effects on student attitudes toward equal rights, indicating the need to consider both universal and context-specific theoretical frameworks.

In Chap. 5, Villalobos, Treviño, Wyman and Béjares set out to disentangle the effects of one of these school composition variables, which is also one of the most relevant sources of diversity in education nowadays: namely segregation of immigrant students. They paid particular attention to the strategies that educational systems employ to address this type of diversity and discussed in depth the issue of educational segregation of immigrant students within the education system. Focusing on the distribution, concentration and spread of immigrant students among schools and countries, Villalobos et al. aimed to understand how education systems generate mechanisms to include (or exclude) these students. The analyses described, from a comparative perspective, patterns of segregation in different educational systems and related them to student attitudes toward equal rights for immigrants. In addition to supporting similar findings signaled in Chap. 4, Villalobos et al. confirmed that individual student background characteristics (such as socioeconomic status) were stronger predictors of student attitudes of tolerance toward equal rights than school composition indicators. Moreover, country specific and differential effects highlight the importance of context showing, for example, that the level of segregation of immigrant students in schools is a predictive factor only in a few contexts and that the relationships between this factor and student egalitarian attitudes (although largely negative and moderate in magnitude) can be both negative and positive.

In Chap. 6, Carrasco and Irribarra focused on the importance of the school environment for the development of egalitarian attitudes. More specifically, they argued the importance of stimulating open classroom discussion, in which free dialogue and critical debate are encouraged among people of diverse backgrounds. Although the importance of an open classroom climate in the development of attitudes toward equal rights (mainly toward immigrants) is largely established by previous research, the work presented in this chapter makes a unique contribution to the field by: (a) demonstrating an appropriate treatment for individual and (aggregated) school-level measures of open classroom climate, (b) providing consideration of multiple measures of tolerance in relation to open classroom climate, and (c) providing estimations of both main and moderation effects. Their results confirm that, across countries, the level of openness to the discussion of political and social issues in classrooms during regular lessons was systematically related to student attitudes toward equal rights for woman, all ethnic groups and immigrants. Moderation effects are, nevertheless, context specific. More specifically, only one country (Austria) showed that an open classroom climate had a booster effect over students clustered in schools with disadvantaged student populations in terms of socioeconomic backgrounds.

In Chap. 7, Miranda, Castillo and Cumsille looked in-depth at individual background characteristics by testing whether young people with greater 
socioeconomic resources showed more egalitarian attitudes. More specifically, they focused on determining the extent to which attitudes toward equal rights for immigrants, ethnic groups and women differed according to socioeconomic background and gender. In agreement with previous studies (Barber et al. 2013; Janmaat 2014; Dotti Sani and Quaranta 2017), they found that students socialized in homes with lower resources showed less support for equal rights for immigrants, ethnic minorities and women. However, unlike previous approaches, they were also able to shed some light on variations in these relationships between different groups of young people and between different socioeconomic measures, finding, for example, that girls and students from an immigrant background tended to be more in favor of equal rights. They also found that some measures of socioeconomic status (parental education and books at home) showed stronger associations with egalitarian attitudes for specific groups (girls), and that cross-country variation indicated country-specific patterns in relationships.

\section{Main Conclusions:}

- International large-scale assessments, such as ICCS 2009, have the potential to tremendously improve the study of tolerance in youth, by providing the opportunity to analyze differing explanatory mechanisms in a multitude of multi-leveled contexts.

- Tolerance is a controversial, multifaceted and complex concept. In a restricted sense, in educational settings, tolerance can be conceptualized and empirically studied in terms of attitudes toward equal rights for three different social groups: immigrants, ethnic minorities and women.

- A broader conceptualization of tolerance (in terms of attitudes toward equal rights for immigrants, ethnic groups and women) can be applied to ICCS 2009 data, and the operationalization of the concepts developed in this report are universally relevant and comparable across a large number of cultural and educational settings.

- Attitudes toward equal rights for different social groups may be developed in harmony. Young people who are willing to support equal rights for immigrants and ethnic groups are also willing to endorse equal rights for women.

- Gender differences (favoring girls) in young people's egalitarian attitudes are notable. Girls tend to have more positive attitudes toward equal rights than boys. The positive attitudes of girls go beyond a mere self-interested demand. Girls do not only show higher endorsement of gender equality but also more positive attitudes toward equal rights for immigrants and ethnic minorities.

- Individual background student characteristics show a (relatively) stronger association with attitudes toward equal rights than school factors. In particular, the socioeconomic status of young people and their levels of 
interest in social and political issues show strong links with students' attitudes toward equal rights.

- Democratic school cultures and school climates that nurture classroom discussion and encourage free dialogue and critical debate among people of diverse backgrounds show particularly strong links with positive student attitudes toward equal rights. These relationships are largely consistent across educational settings around the world.

- Both universal and context-specific explanatory mechanisms must be considered when identifying factors and conditions that may help schools and teachers to promote tolerance.

\subsection{Discussion and Implications}

We argue that international comparative studies, such as ICCS 2009, provide opportunities to study tolerance in youth. Analysis of such data may reveal differing explanatory mechanisms in a multitude of multi-levelled contexts.

We sought to confirm extant research and identify the factors and conditions that have the potential to help schools and teachers promote tolerance. We focused on gathering evidence on: (a) broader conceptualizations of tolerance toward traditionally disadvantaged groups, including attitudes toward the rights of immigrants, ethnic groups and women, (b) the potential relationships among attitudes of tolerance toward equal rights for different groups, (c) the strength of relationships between egalitarian attitudes and variables measured at different levels (such as the individual, school, or educational system level), (d) the complexity of direct and indirect (moderated) relationships, and (e) the variation of these relationships across countries (common and country-specific differential effects).

Our conceptualization of tolerance included attitudes toward the rights of three different social groups: immigrants, ethnic minorities and women. In previous work using the ICCS 2009 data, Schultz (2015) highlighted the importance of looking at additional measures of attitudes toward equal rights; next to attitudes toward immigrants, Schultz showed the value of also examining attitudes toward equal rights for ethnic minorities. Higdon (2015) followed a similar approach, demonstrating the potential need to also consider attitudes toward women's rights. The analyses in Chap. 3 make an important contribution to the literature by simultaneously taking into account all three measures and extending the analyses from a subset of countries to all 38 countries included in ICCS 2009. Miranda and Castillo thus provide conclusive empirical support for a broader conceptualization of tolerance based on 
the ICCS 2009 data, justifying not only the analyses presented in this report but also future comparative research on the topic.

Chapter 3 also indicates a clear interconnection between the three measures of tolerance, with positive associations ranging from 0.39 to 0.95 across measures and countries. Specifically, Miranda and Castello found that young people who were willing to support equal rights for immigrants and ethnic groups were also willing to endorse equal rights for women. Although this may seem an intuitive expectation, the strength or direction of such associations should not be taken for granted. ${ }^{1}$

This finding is extremely important for various formal and informal educational channels, because it suggests that positive attitudes toward equal rights for others may be developed in harmony.

Moreover, in Chaps. 4 and 7, the analyses revealed that girls tend to have more positive attitudes toward equal rights than boys and that the more positive attitudes of girls go beyond a mere self-interested demand, as their attitudes were not only related to gender equality but also to more positive attitudes toward equal rights for immigrants and ethnic minorities.

This in turn suggests the importance of replicating such findings in adult populations. If research confirmed similar results in an adult population, this would prompt questions such as: Would there be an increase the equal rights if women received the same political representation as men? Although the gender balance in decision-making positions has been steadily improving around the world, in most countries women are still underrepresented in political systems. For instance, in 2017 only $23 \%$ of the seats in national parliaments were occupied by women (Inter-Parliamentary Union 2017). Considering this, the equalization of political representation may be a powerful mechanism to promote the development of democratic principles both in schools and wider society. Furthermore, future research on this issue may reveal links between the implementation of democratic principles and more egalitarian representation of disadvantaged groups.

Chapters 4-7 examined the factors and conditions at different levels (especially student and school levels) that exhibited positive relationships with young peoples' attitudes toward equal rights for immigrants, ethnic groups and women. Chapters 4-6, in particular, demonstrated the (relatively) stronger effect of individual variables over school factors. Nevertheless, in agreement with previous research, both individual and school characteristics were found to be consistent (for the majority of countries and for all three tolerance measures included in the analyses) predictors of positive attitudes toward equal rights. Strong and consistent links with all tolerance measures were found for individual background student characteristics such as socioeconomic background (Chaps. 5 and 7) and student interest in social and political issues (Chap. 4). When considering school characteristics, the majority of our research reaffirmed the importance of democratic school cultures and the

\footnotetext{
${ }^{1}$ Previous research (Barber et al. 2013; Isac 2015a) showed weak links and even negative relationships between other attitudinal civic outcomes measured in ICCS 2009.
} 
beneficial effects of a school climate that nurtures open classroom discussion and encourages free dialogue and critical debate among people of diverse backgrounds (see Chap. 6). Other individual and school factors demonstrated differing associations that were group or country dependent. For example, the research in Chap. 7 highlighted differences in the strength of the association between different socioeconomic measures and attitudes toward equal rights for different groups of students (girls, immigrants). Chapters 4 and 5 showed that school composition variables (for example, the level of segregation of immigrant students in schools) showed strong country-specific differential effects on egalitarian attitudes. Chapter 6 confirmed that moderation effects, while worth investigating, are only relevant in a few specific contexts.

Combining these findings may provide useful guidance for educators and policymakers in designing school interventions aimed at promoting tolerance. Our research suggests that more attention (e.g. targeted interventions) should be paid to vulnerable and disadvantaged students (in terms of socioeconomic background, levels of knowledge and interest) and schools that serve disadvantaged student populations.

As the most promising school strategy, the research largely reinforces the importance of democratic school cultures. Encouraging students to discuss controversial issues and allowing them to make up their own minds while presenting several sides to the issues under discussion seems to be a promising teaching practice. Therefore, school interventions coupled with continuous teacher development programs aimed at promoting higher levels of open classroom discussion may prove effective. Nevertheless, as Carrasco and Irribarra mentioned in Chap. 6, teachers often find it difficult to engage in such discussion and, in many countries, they (as well as school leaders) indicate a strong need for professional development in this area (Van Driel et al. 2016). The need for the teacher to balance classroom discussion which is inclusive of different views, while also participating in those discussions with a personal position on the issues under discussion, requires not only high levels of teaching skill but also the confidence and freedom to tackle issues, including those which are potentially controversial in nature. Whole-school approaches involving institutional support from school authorities, the school community and parents may be crucial to helping teachers to introduce with confidence the discussion of controversial issues involving ethnicity, immigration and gender to the classroom.

The studies also indicate large heterogeneous findings across countries, pointing to the need to take into account the specificity of contexts and the differential effect that some school characteristics may have. Depending on the country context, heterogeneous school composition, or different levels of segregation of immigrant students may or may not create conditions for the development of egalitarian attitudes. It is also important to acknowledge that immigrant students are not a monolithic group. Depending on the country of destiny and specific family 
situations, they can come from affluent contexts or from the most disadvantaged groups in society (Engel et al. 2014; Hastedt 2016). The same holds true for the potential compensatory effects of open classroom climates over students clustered in schools with disadvantaged socioeconomic backgrounds. Potential interventions aimed at promoting tolerance should include careful analyses of settings, which may provide further information on conditions for promoting success.

\section{Recommendations for Educators and Policymakers:}

- Support interventions targeted at vulnerable youth (for example disadvantaged students in terms of socioeconomic status, levels of knowledge and interest) and schools that serve disadvantaged student populations.

- Encourage school interventions coupled with continuous teacher development programs aimed at promoting democratic school cultures and climates.

- Support teachers with continuous professional development, building the skills needed to address diversity and promote tolerance.

- Involve and support collaboration between wider groups of stakeholders (for example, teachers, school authorities, parents, and local communities).

- Carry out careful analyses of local settings to gain insights into context-specific conditions for promoting tolerance.

\subsection{Limitations and Avenues for Future Studies}

International studies, such as ICCS 2009, are invaluable resources for the study of tolerance in youth. Applying state-of-the-art methodological approaches to the rich data collected by ICCS 2009 has enabled us to address some interesting questions. Although cross-sectional large-scale assessment surveys may have their limitations, our view is that these are also opportunities for further research in the field.

Firstly, due to the cross-sectional nature of ICCS, we acknowledge that no causal inferences can be drawn from the results reported here. Therefore, we advise the reader to interpret the findings rather as starting points for discussion, formulating hypotheses and establishing patterns; hypotheses can be subsequently tested using rigorous interventions and impact evaluations. Similarly, complementary methodological approaches (such as qualitative case studies) may be adopted to disentangle the mechanisms causing the patterns identified in the data.

Secondly, as mentioned in Chap. 1, the concept of tolerance investigated here is complex and multifaceted. Although this report makes an important contribution to the field by considering multiple measures of tolerance and documenting their 
validity in several educational systems, broader conceptualizations may be thought of when designing future research, including future international large-scale assessments of this kind. More specifically, the measure of tolerance may be further improved to capture not only political but also social tolerance (Weldon 2006; Quintelier and Dejaeghere 2008), attitudes of tolerance toward a wider range of groups (Caro and Schulz 2012; Forst 2003; Green et al. 2006; Mutz 2001), while perspectives oriented toward rejection of social groups (intolerance, discrimination) may be particularly useful for identifying youth at risk.

Lastly, this report has tackled the complexities inherent in the study of relationships between differing classroom factors and student attitudes toward equal rights. Among such issues, we note that the ICCS 2009 was designed to sample one intact class per selected school in most countries. For this reason, school and classroom levels are confounded. Therefore, results concerning the relevance of school factors in relation to attitudes toward equal rights must be interpreted with caution. Future research could address the need to better differentiate both theoretically and empirically between school and class-related factors and their relationship to young peoples' egalitarian attitudes (using sampling strategies targeting more than one class per school). Complex methodological and theoretical questions pose further aspects for reflection, including identifying the most appropriate measurement models for students' ratings of classrooms, addressing plausible endogeneity in modelling multilevel data, and unfolding the complex explanatory mechanisms (the why, how, and when) that link classroom factors to student attitudes (see Stapleton et al. 2016).

\section{Recommendations for Further Research:}

- Interpret correlational findings only as starting points for discussion, formulating hypotheses and establishing patterns.

- Complement knowledge acquired through correlational studies with rigorous impact evaluations and qualitative studies.

- Develop even broader conceptualizations of tolerance that take into account its complex and multifaceted nature.

- Address the need to better differentiate both theoretically and empirically between school- and class-related factors that are related to tolerant attitudes.

- Reflect on complex methodological and theoretical questions, such as identifying the most appropriate measurement models for students' ratings of classrooms and addressing plausible endogeneity in modeling multilevel data.

- Unfold the complex explanatory mechanisms (the why, how and when) that link classroom factors to student attitudes. 


\section{References}

Barber, C., Fennelly, K., \& Torney-Purta, J. (2013). Nationalism and support for immigrants' rights among adolescents in 25 countries. Applied Developmental Science, 17(2), 60-75.

Caro, D., \& Schulz, W. (2012). Ten hypotheses about tolerance toward minorities among Latin American adolescents. Citizenship, Social and Economics Education, 11(3), 213-234. https:// doi.org/10.2304/csee.2012.11.3.213.

Desa, D. (2014). Evaluating measurement invariance of TALIS 2013 complex scales. Retrieved from http://www.oecd-ilibrary.org/education/evaluating-measurement-invariance-of-talis2013-complex-scales_5jz2kbbvlb7k-en.

Dotti Sani, G. M., \& Quaranta, M. (2017). The best is yet to come? Attitudes toward gender roles among adolescents in 36 countries. Sex Roles, 77, 30-45. https://doi.org/10.1007/s11199-0160698-7.

Engel, L. C., Rutkowski, L., \& Rutkowski, D. (2014). Global mobility and rising inequality: A cross-national study of immigration, poverty, and social cohesion. Peabody Journal of Education, 89(1), 123-140. https://doi.org/10.1080/0161956X.2014.862480.

Forst, R. (2003). Toleration, justice and reason. In C. McKinnon \& D. Castiglione (Eds.), The culture of toleration in diverse societies (pp. 71-85). Manchester, UK: Manchester University Press.

Green, A., Preston, J., \& Janmaat, J. (2006). Education, equality and social cohesion: A comparative analysis. Hampshire, UK: Palgrave Macmillan.

Hastedt, D. (2016). Mathematics achievement of immigrant students. Cham, Switzerland: Springer International Publishing. https://doi.org/10.1007/978-3-319-29311-0.

Higdon, J. D. (2015). Measuring and modeling intercultural attitudes among adolescents across Europe: A multi-level, multiple-group analysis examining student attitudes, intergroup contact, and school climate (Ph.D. thesis). Harvard Graduate School of Education, CT, USA. Retrieved from: http://nrs.harvard.edu/urn-3:HUL.InstRepos:16461055.

Inter-Parliamentary Union. (2017). PARLINE database on national parliaments. Retrieved from http://www.ipu.org/wmn-e/world.htm.

Isac, M. M. (2015a). Effective civic and citizenship education: A cross-cultural perspective (Ph.D. thesis). University of Groningen, Groningen, The Netherlands. Retrieved from https://www. rug.nl/research/portal/files/23847395/Complete_thesis.pdf.

Isac, M. M. (Ed.). (2015b). Tolerance through education: Mapping the determinants of young people's attitudes towards equal rights for immigrants and ethnic/racial minorities in Europe. Luxembourg: Publications Office of the European Union.

Janmaat, J. G. (2014). Do ethnically mixed classrooms promote inclusive attitudes towards immigrants everywhere? A study among native adolescents in 14 western countries. European Sociological Review, December 2014, 810-822.

Mutz, D. C. (2001). Tolerance. In N. J. Smelser \& P. B. Baltes (Eds.), International encyclopedia of the social and behavioral sciences (pp. 15766-15771). Amsterdam: Elsevier.

Quintelier, E., \& Dejaeghere, Y. (2008). Does European citizenship increase tolerance in young people? European Union Politics, 9(3), 339-362.

Rutkowski, L., \& Svetina, D. (2014). Assessing the hypothesis of measurement invariance in the context of large-scale international surveys. Educational and Psychological Measurement, 74 (1), 31-57. https://doi.org/10.1177/0013164413498257.

Schultz, W. (2015). A question of perspective? Measuring views on equal rights and opportunities among minority groups in European large-scale surveys. In M. M. Isac (Ed.), Tolerance through education: Mapping the determinants of young people's attitudes towards equal rights for immigrants and ethnic/racial minorities in Europe. Luxembourg: Publications Office of the European Union. Retrieved from https://ec.europa.eu/jrc.

Sherrod, L. R., \& Lauckhardt, J. (2009). The development of citizenship. In R. M. Lerner \& L. Steinberg (Eds.), Handbook of adolescent psychology (pp. 372-407). Hoboken, NJ: Wiley. 
Stapleton, L. M., Yang, J. S., \& Hancock, G. R. (2016). Construct meaning in multilevel settings. Journal of Educational and Behavioral Statistics, 41(5), 481-520. https://doi.org/10.3102/ 1076998616646200.

Van Driel, B., Darmody, M., \& Kerzil, J. (2016). Education policies and practices to foster tolerance, respect for diversity and civic responsibility in children and young people in the EU. NESET II report. Luxembourg: Publications Office of the European Union. https://doi.org/10. $2766 / 46172$.

Weldon, S. A. (2006). The institutional context of tolerance for ethnic minorities: A comparative, multilevel analysis of Western Europe. American Journal of Political Science, 50(2), 331-349.

Open Access This chapter is licensed under the terms of the Creative Commons Attribution-NonCommercial 4.0 International License (http://creativecommons.org/licenses/by-nc/ 4.0/), which permits any noncommercial use, sharing, adaptation, distribution and reproduction in any medium or format, as long as you give appropriate credit to the original author(s) and the source, provide a link to the Creative Commons license and indicate if changes were made.

The images or other third party material in this chapter are included in the chapter's Creative Commons license, unless indicated otherwise in a credit line to the material. If material is not included in the chapter's Creative Commons license and your intended use is not permitted by statutory regulation or exceeds the permitted use, you will need to obtain permission directly from the copyright holder.

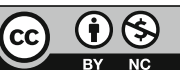

